\title{
Response to lenalidomide in myelodysplastic syndromes
}

The cytotoxic effects of lenalidomide could be used as a surrogate marker of achieving a transfusion independent response in patients with myelodysplastic syndromes (MDS), according to a recent study. "This is the first study to analyze the relationship between treatment-related cytopenias and response in patients with MDS," comments the corresponding author of the study.

MDS comprise a spectrum of bone marrow disorders that are associated with cytopenias and thereby promote an increased risk of bleeding and infection. In the US, more than 10,000 people per year are affected by MDS, and cure can only be achieved by bone-marrow transplantation. However, as the median age of diagnosis is over 70 years, most patients receive supportive care with blood transfusions, growth factor and non-growth-factor therapies. Lenalidomide is an immunomodulatory agent that has proved particularly effective for treating patients with deletion $5 \mathrm{q}$ abnormalities, with $67 \%$ of such patients achieving transfusion independence. Half of patients with MDS treated with lenalidomide, however, experience grade 3 or 4 neutropenia or thrombocytopenia and require dose reduction for myelosuppresion.

Mikkael Sekeres et al. investigated whether lenalidomide-induced cytopenias could serve as a surrogate marker for transfusion independent response to the drug. They assessed 362 patients who were enrolled into two phase II studies (MDS002 and MDS-003) and who had low-risk MDS and were transfusion dependent with or without the deletion $5 \mathrm{q}$ cytogenetic abnormality.

\section{4 ...therapy-related cytopenias} may be a marker of subsequent response... 77

Patients harboring the deletion $5 \mathrm{q}$ abnormality developed more treatment-related thrombocytopenia than those without the abnormality. Achieving transfusion independent response was correlated with development of thrombocytopenia for patients with the $5 q$ deletion regardless of the baseline platelet count. Among patients with the $5 \mathrm{q}$ deletion, $70 \%$ whose platelet count decreased by $\geq 50 \%$ achieved transfusion independence. This was significantly higher than the transfusion independent response seen in those whose platelet count remained stable-only $42 \%$ of these patients achieved a response. Treatment-related thrombocytopenia and transfusion independence did not correlate with patients who lacked the $5 \mathrm{q}$ deletion. For patients without neutropenia at baseline, $82 \%$ who had an absolute neutrophil count that decreased by $\geq 75 \%$ showed a transfusion independent response, compared with $51 \%$ of those whose absolute neutrophil count remained the same or decreased by less than $75 \%$.

"This research advises treating physicians that therapy-related cytopenias may be a marker of subsequent response, and not necessarily a reason to discontinue therapy prematurely," says Dr Sekeres. "This study shows that those with low-risk MDS with a $5 q$ deletion and a $75 \%$ reduced absolute neutrophil count or platelet count decrease of $50 \%$ or more in response to lenalidomide achieved transfusion independence." Thus, lenalidomide-induced cytopenias early in the course of treatment could be used as a surrogate marker for response to therapy and predictive of a transfusion response. Dr Sekeres commented that "the implications for this research are that the dose of lenalidomide, or other MDS drugs, could be titrated to a degree that correlates with suppression of the malignant clone."

\section{Lisa Hutchinson}

Original article Sekeres, M. A. et al. Relationship of treatment-related cytopenias and response to lenalidomide in patients with lower-risk myelodysplastic syndromes. J. Clin. Oncol. 26, 5943-5949 (2008). 